\title{
Induced superconductivity in the three-dimensional topological insulator $\mathrm{HgTe}$
}

\author{
Luis Maier, ${ }^{1}$ Jeroen B. Oostinga,, , * Daniel Knott, ${ }^{1}$ Christoph Brüne, ${ }^{1}$ Pauli Virtanen, ${ }^{2}$ Grigory \\ Tkachov, ${ }^{2}$ Ewelina M. Hankiewicz, ${ }^{2}$ Charles Gould, ${ }^{1}$ Hartmut Buhmann, ${ }^{1}$ and Laurens W. Molenkamp ${ }^{1}$ \\ ${ }^{1}$ Physikalisches Institut (EP3), University of Würzburg, Am Hubland, D-97074, Würzburg, Germany \\ ${ }^{2}$ Institut für Theoretische Physik und Astrophysik (TP4), \\ University of Würzburg, Am Hubland, D-97074, Würzburg, Germany
}

(Dated: October 10, 2018)

\begin{abstract}
A strained and undoped $\mathrm{HgTe}$ layer is a three-dimensional topological insulator, in which electronic transport occurs dominantly through its surface states. In this Letter, we present transport measurements on HgTe-based Josephson junctions with $\mathrm{Nb}$ as superconductor. Although the NbHgTe interfaces have a low transparency, we observe a strong zero-bias anomaly in the differential resistance measurements. This anomaly originates from proximity-induced superconductivity in the HgTe surface states. In the most transparent junction, we observe periodic oscillations of the differential resistance as function of an applied magnetic field, which correspond to a Fraunhofer-like pattern. This unambiguously shows that a precursor of the Josephson effect occurs in the topological surface states of HgTe.

PACS numbers: 73.23.-b, 73.25.+i, 72.80.Ey, 74.45.+c
\end{abstract}

Topological insulators (TIs) are a recently discovered new class of materials [1]. In two dimensions (2-D), a TI is an insulating material with conducting helical states at its edges. The first experimental realization of a 2-D TI was a HgTe quantum well, in which the topological state of matter was unambiguously demonstrated by the observation of the quantum spin Hall effect in electronic transport experiments [2]. The first three-dimensional (3-D) TIs, $\mathrm{Bi}_{1-x} \mathrm{Sb}_{x}, \mathrm{Bi}_{2} \mathrm{Se}_{3}$ and $\mathrm{Bi}_{2} \mathrm{Te}_{3}$, were discovered soon after [3]. 3-D TIs are bulk insulators with conducting states at their surfaces. Since these surface states are effectively described by Dirac Hamiltonians, the corresponding quasiparticles behave as chiral Dirac fermions, i.e. the spin and momentum of the electrons are locked [1].

Recently, it has been predicted that, due to the superconducting proximity effect, a Dirac fermion at the surface of a 3-D TI can be split into two so-called Majorana quasiparticles [4]. These are zero-energy bound states that may be present at the surface when a TI is contacted by a superconductor. The realization of a superconductor-topological insulator-superconductor (STI-S) junction is an important first step to study the existence of Majorana fermions. S-TI-S junctions based on $\mathrm{Bi}_{2} \mathrm{Se}_{3}$ [5] and $\mathrm{Bi}_{2} \mathrm{Te}_{3},[$ ] have been recently reported and a Josephson supercurrent has been observed in both these 3-D TIs. However, since the Bi-based 3-D TIs have a large density of states in their bulk, only a small part of the observed supercurrent can be attributed to superconducting correlations in the surface states. We recently demonstrated that strained HgTe is a 3-D TI with negligible bulk conductance when the Fermi level is inside its bandgap [7]. This material is thus a more promising candidate to study the Josephson effect in S-TI-S junctions without being hindered by bulk states.

In this Letter, we study electronic transport through
S-TI-S junctions based on superconducting Nb wires contacted to a strained HgTe layer, in which the electrical conduction is dominated by surface state transport. Although it turns out that the $\mathrm{Nb}-\mathrm{HgTe}$ interfaces have a low transparency, we clearly observe induced superconductivity and transport through surface states approaching the supercurrent regime. Our results provide clear evidence of superconducting correlations in the topological surface states of $\mathrm{HgTe}$.

Two bulk HgTe layers of 50 and $70 \mathrm{~nm}$ thickness, respectively, are grown by molecular beam epitaxy on a CdTe substrate. The samples are etched in an Ar plasma to obtain $2 \mu \mathrm{m}$ wide $\mathrm{HgTe}$ stripes. On the top surface of the $\mathrm{HgTe}$ stripes, $68 \mathrm{~nm}$ thick $\mathrm{Nb}$ superconducting wires are deposited by using ultra high vacuum sputtering and lift-off techniques. Each pair of closely-spaced neighboring wires forms an S-TI-S junction (Figs. 1a, b). The transport measurements on these devices have been performed at dilution refrigerator temperatures. We have measured the differential resistance of the junctions as function of magnetic field and current bias by using standard lock-in detection techniques. Here we will discuss the data of two of the closest junctions with $150 \mathrm{~nm}$ contact spacing; other junctions show consistent behaviour.

In a first set of measurements we investigate the superconductiong properties of the $50 \mathrm{~nm}$ thick HgTe layer. In order to characterize the transport properties of this bulk $\mathrm{HgTe}$ layer, we have fabricated from the same wafer as used for one of the S-TI-S junctions, a large six-terminal Hall bar with a channel length of $600 \mu \mathrm{m}$ and width of $200 \mu \mathrm{m}$ (similar to the device reported in Ref. 7] ). From the longitudinal and transversal magnetoresistance measurements at $T=4.2 \mathrm{~K}$, we extract an electron density of $n_{e} \approx 5 \cdot 10^{11} \mathrm{~cm}^{-2}$ and a mobility of $\mu_{e} \approx 3 \cdot 10^{4} \mathrm{~cm}^{2} /$ Vs. These values are typical for strained HgTe layers in which electronic transport is dominantly through their Dirac 

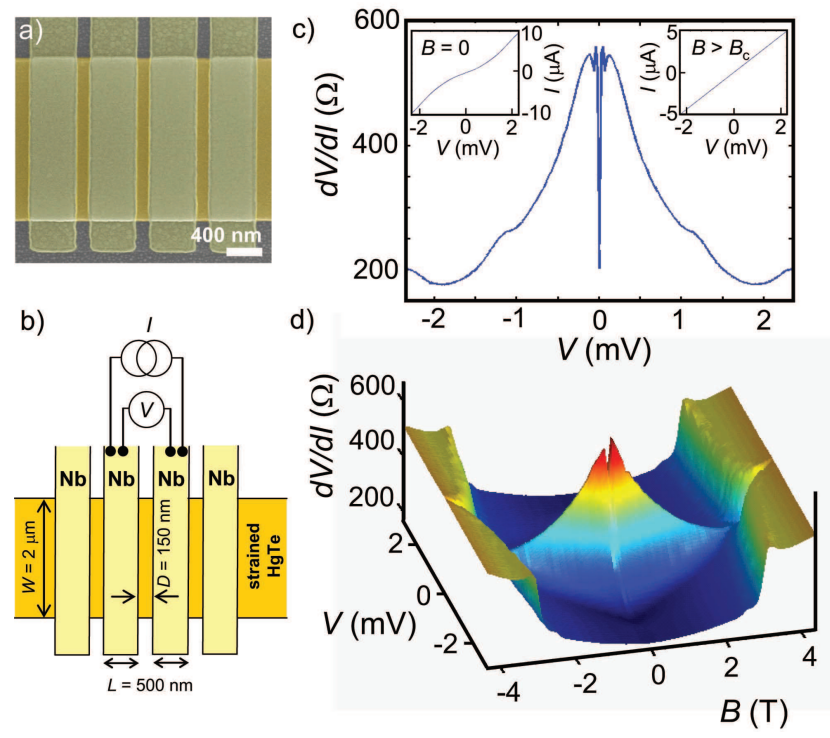

FIG. 1: (a) Scanning electron microscopy image of an etched $\mathrm{HgTe}$ stripe contacted by four Nb wires. (b) Schematic picture of three Nb-HgTe-Nb junctions. (c) $d V / d I$ as function of measured voltage across one of the junctions $(B=0)$. Insets show $I-V$ characteristics at $B=0$ (left) and $B>B_{c}$ (right). (d) $d V / d I$ versus $V$ and applied $B$.

surface states [7]. Moreover, at magnetic fields higher than $2 \mathrm{~T}$ we observe a series of quantization steps in the Hall resistance, indicating that transport is indeed occurring through 2-D states [8, 9 ].

Since the transport is dominated by surface states, it is possible to investigate the behavior of a S-TI-S junction, fabricated on this same $50 \mathrm{~nm}$ thick layer. In order to study transport in the superconducting regime, we have performed two-terminal measurements at a base temperature of $T \approx 25 \mathrm{mK}$ (which is far below the experimentally verified critical temperature of $\mathrm{Nb}, T_{c} \approx 9 \mathrm{~K}$ ). Since each Nb terminal is split into two leads - one for current injection and the other for voltage measurement - we can simultaneously measure the differential resistance of the junction and the electrochemical potential difference between the $\mathrm{Nb}$ contacts (Fig. 1b). The differential resistance $(d V / d I)$ as a function of the measured voltage across the junction $(V)$ provides detailed information about the energy dependence of the transmission probability of quasiparticles through the junction [10].

Fig. 1c shows $d V / d I$ as function of $V$ for one of the junctions at zero magnetic field. At $|V| \approx 2 \mathrm{mV}$, we observe a pronounced dip in $d V / d I$. This is when the potential difference between both $\mathrm{Nb}$ contacts equals twice the superconducting gap $\left(\Delta_{N b} \approx 1 \mathrm{meV}\right)$. When $V= \pm 2 \Delta_{N b} / e$, the upper band edge of the quasiparticle states in one $\mathrm{Nb}$ lead is aligned with the lower band edge of the other (Fig. 2c). At these energies, the quasiparticle density of states in the $\mathrm{Nb}$ as well as the Andreev reflection (AR) probability is enhanced [11], which gives rise to an increased transmission probability at both interfaces, and thus a decrease of the differential resistance of the junction 10.

For $|V| \gtrsim 2 \mathrm{mV}$, when the potential difference between the $\mathrm{Nb}$ contacts is larger than $2 \Delta_{N b} / e$, the differential resistance is $R_{n} \approx 200 \Omega$. This is the normal conductivity transport regime, where the superconducting gaps of the $\mathrm{Nb}$ leads have no energy overlap and single quasiparticles from filled states below the gap in one lead are transmitted to empty states above the gap in the other lead.

If $|V|<2 \Delta_{N b} / e$, the superconducting gaps of the $\mathrm{Nb}$ leads have an energy overlap. In this sub-gap regime, quasiparticles can only be transmitted through the junction by AR processes at the interfaces [11]. Fig. 1c shows that below $|V| \approx 2 \mathrm{mV}$ a strong increase of the differential resistance occurs on lowering the voltage difference between the Nb leads. This indicates that the AR probability is suppressed due to the low transparency of the $\mathrm{Nb}-\mathrm{HgTe}$ interfaces.

A further characterization of the junction is done by measuring the magnetic field dependence of the $d V / d I$ versus $V$ characteristics (Fig. 1d). At perpendicular magnetic fields $(B)$ larger than $3 \mathrm{~T}$, we observe a steep increase in the differential resistance, which is due to the transition of $\mathrm{Nb}$ from superconducting to normal state. If the magnetic field is decreased below $B_{c} \approx 3 \mathrm{~T}$, the superconducting gap opens and the band edge can be probed by measuring the corresponding $d V / d I$ dip at $V= \pm 2 \Delta_{N b} / e$ as function of magnetic field (Fig. 3a). This dip, where $\frac{\partial}{\partial V}(d V / d I)=0$, is visualized in a colorplot by numerically differentiating $d V / d I$ versus $V$ and plotting the obtained absolute value versus $V$ and the applied magnetic field (Fig. 3b). The figures clearly show that the gap increases from 0 to $\sim 1 \mathrm{meV}$ when the magnetic field decreases from $\sim 3$ to $0 \mathrm{~T}$.

Interestingly, Fig. 1c shows a strong dip in $d V / d I$ at very small bias. Figs. 3c, d show that this zero-bias anomaly (ZBA) appears at $|V| \lesssim 100 \mu \mathrm{V}$ and $|B| \lesssim 10$ $\mathrm{mT}$. A ZBA can appear in Josephson junctions due to the superconducting proximity effect in the normal conductor [12]. Also in our S-TI-S junction, Cooper pairs "leak" from the $\mathrm{Nb}$ into the $\mathrm{HgTe}$ below the contacts, yielding electron correlations in the surface states 13 . These correlations give rise to induced superconductivity on an energy scale of the order of the induced superconducting gap $\left(\Delta_{i n d}\right)$ which depends on the transparency of the contacts. The fact that the observed ZBA is very strong indicates the surface character of the induced superconductivity in $\mathrm{HgTe}$ [8]. In conventional materials with bulk transport, the ZBA is usually much weaker due to the decay of the correlations with distance from the superconductor [12]. When the bias across the junction is smaller than twice the induced gap $\left(|V|<2 \Delta_{\text {ind }} / e\right)$, the AR probability at each interface is strongly enhanced (Fig. 2a), yielding a large suppression of the differential resistance. In our junction, the ZBA appears below $|V| \approx$ 


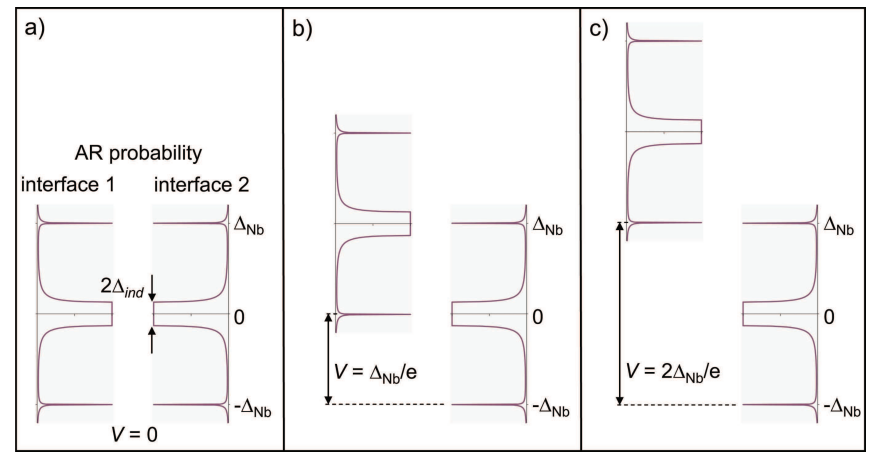

FIG. 2: The appearance of the main features in the characteristics of $d V / d I$ versus $V$ can be explained by considering the AR probabilities at both Nb-HgTe interfaces [8]. The schematic pictures show that the transmission probability through the junction is highest when the AR probability at both interfaces is highest: that is for (a) $|V|=0$, (b) $|V|=\Delta_{N b} / e$, or (c) $|V|=2 \Delta_{N b} / e$. Note that at the band edges $\left( \pm \Delta_{N b}\right)$, the quasiparticle density of states in $\mathrm{Nb}$ is enhanced, leading to an additional increase in transmission probability at these energies.

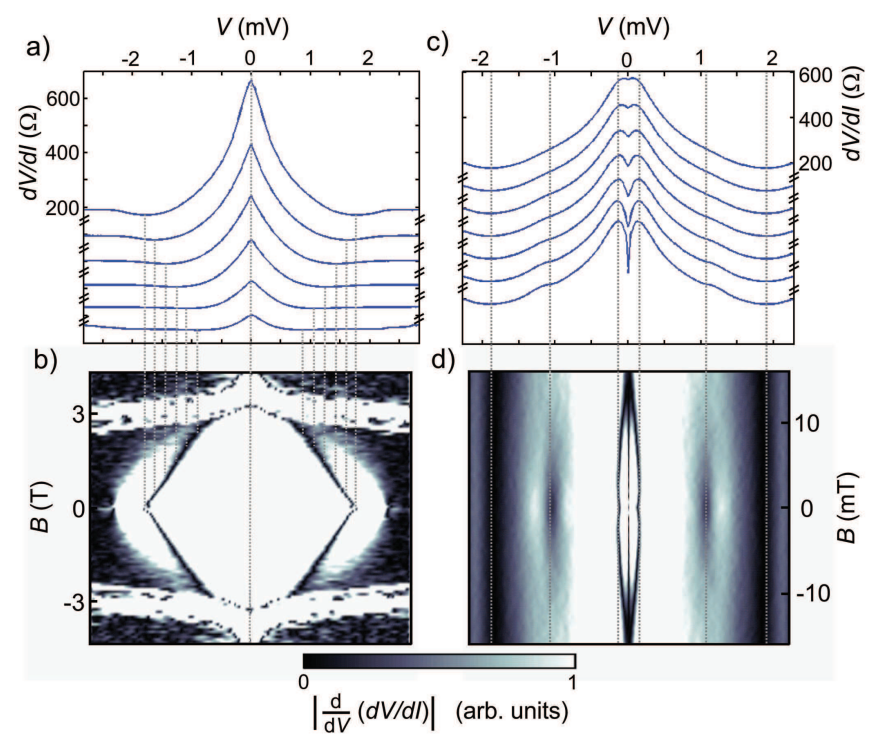

FIG. 3: (a) $d V / d I$ versus $V$ at high magnetic fields (starting from top: $B=0.04 \mathrm{~T}, 0.52 \mathrm{~T}, 1.00 \mathrm{~T}, 1.48 \mathrm{~T}, 2.04 \mathrm{~T}, 2.52 \mathrm{~T}$; the curves are shifted with respect to the top curve for clarity). (b) Colorplot of the numerically derived data of $\left|\frac{d}{d V}(d V / d I)\right|$ versus $V$ and $B$. (c) $d V / d I$ versus $V$ at low magnetic fields (starting from top: $B=12.2 \mathrm{mT}, 10.2 \mathrm{mT}, 8.3 \mathrm{mT}, 6.4 \mathrm{mT}$, $4.5 \mathrm{mT}, 2.6 \mathrm{mT}, 0.6 \mathrm{mT}$; the curves are shifted with respect to the top curve for clarity). (d) Colorplot of the numerically derived data of $\left|\frac{d}{d V}(d V / d I)\right|$ versus $V$ and $B$.

$100 \mu \mathrm{V}$ (Figs. 4a, b), indicating an induced superconducting gap of $\Delta_{\text {ind }} \approx 50 \mu \mathrm{eV}$, which is close to a theoretically expected value for the topological surface states of $\mathrm{HgTe}$ [8].

The ZBA completely disappears when a perpendicular magnetic field of $|B| \gtrsim 10 \mathrm{mT}$ is applied. A magnetic a)

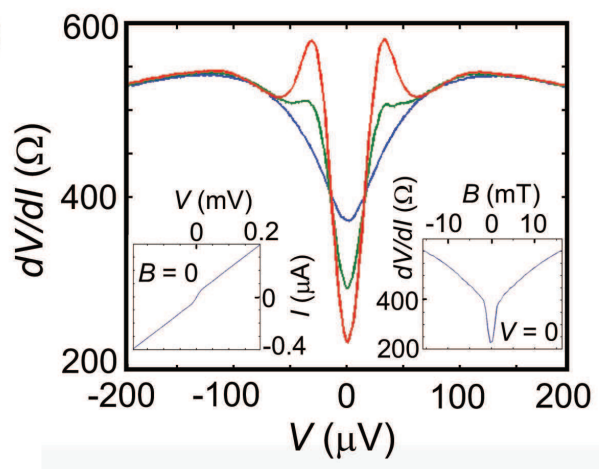

b)

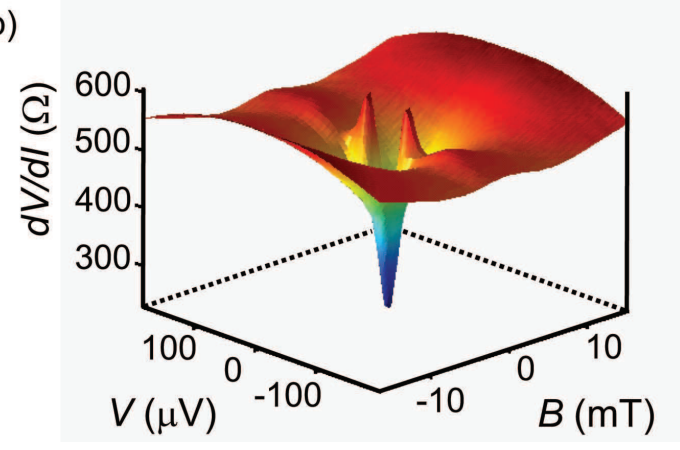

FIG. 4: (a) Low-bias characteristics of $d V / d I$ versus $V$ at $B=0 \mathrm{mT}$ (red), $0.5 \mathrm{mT}$ (green) and $1.0 \mathrm{mT}$ (blue). Left inset shows the $I-V$ characteristic at $B=0$. Right inset shows $d V / d I$ versus $B$ at $V=0$. (b) $d V / d I$ versus $V$ and $B$.

field gives rise to a phase gradient in the order parameter and induces screening currents in the $\mathrm{Nb}$ [14], as has been previously observed in $\mathrm{Nb} / \mathrm{InAs}$ Josephson junctions [15]. The moving condensate leads to momentum and energy transfer in AR processes, resulting in a Doppler shift of the energy of an Andreev reflected quasiparticle with respect to the incoming quasiparticle. When this Doppler shift becomes comparable to $\Delta_{\text {ind }}$ the ZBA will disappear [14]. In our junction, this occurs at a magnetic field value of $\sim 10 \mathrm{mT}$ (Figs. 3c, d and 4b), which is of the same order as the estimated value obtained from calculations [8].

When we consider the sub-gap regime of the $d V / d I$ versus $V$ characteristic in Fig. 1c more carefully, we observe a feature at $|V| \approx 1 \mathrm{mV}$. Its position corresponds approximately to the value of the superconducting gap in $\mathrm{Nb}\left(\Delta_{N b} \approx 1 \mathrm{meV}\right)$. At this bias voltage, the band edge of one $\mathrm{Nb}$ lead is aligned with the center of the gap of the other lead. This alignment leads to an increase of the transmission probability through the junction due to the enhanced AR probability at both interfaces (Fig. 2b). The observed kink in $d V / d I$ at $V= \pm \Delta_{N b} / e$ is therefore closely related to the ZBA. Fig. 3c, d show that this feature indeed disappears at a magnetic field larger than $\sim 10 \mathrm{mT}$, similar to the scale associated with the disappearance of the ZBA.

So far, we have shown that the $\mathrm{Nb}$ contacts induce 
a)

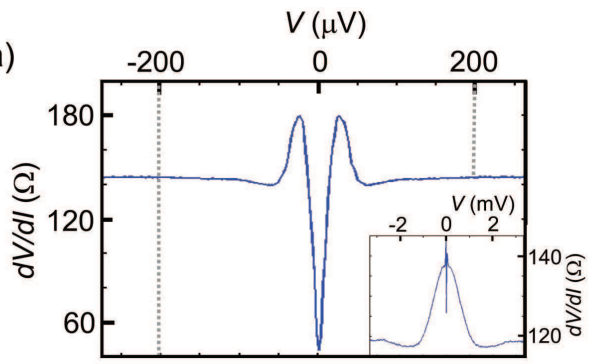

b)

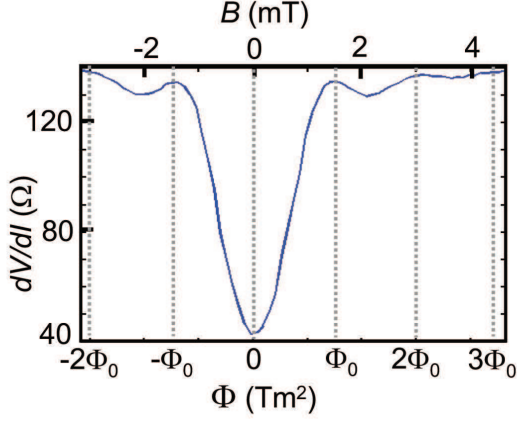

c)

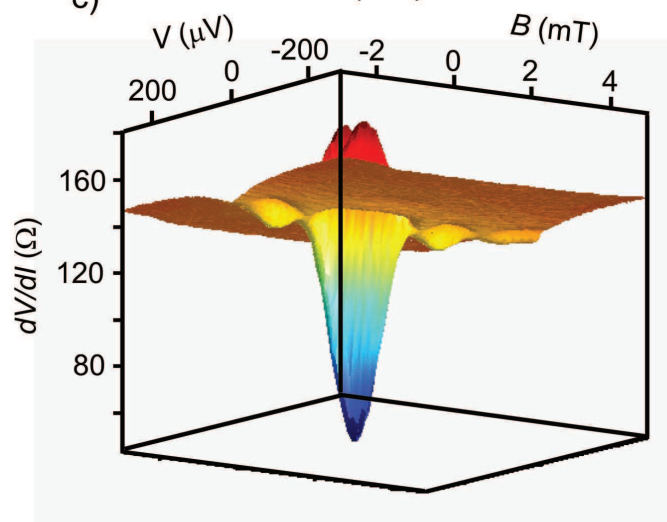

FIG. 5: (a) $d V / d I$ versus $V$ for a second junction with more transparent Nb-HgTe interfaces. (b) $d V / d I$ as function of $B$ at $V=0$. (c) $d V / d I$ as function of $V$ and $B$.

superconductivity in this $\mathrm{HgTe}$ system. These electron correlations occur at least below each contact. If electron transport through the $\mathrm{HgTe}$ between both $\mathrm{Nb}$ contacts is phase coherent, the Josephson effect should give rise to bound states through which a supercurrent can flow. The critical value of such a current $\left(I_{c}\right)$ depends on the phase difference between the condensates in both Nb layers [16]. We do not, however, observe a dissipationless current, indicating that it is destroyed by phase decoherence mechanisms. A supercurrent is absent if the thermal energy of the electromagnetic noise is larger than the Josephson energy [10], which can occur when the wires leading to the sample are not sufficiently filtered and shielded [17]. Given the incomplete filtering in our present setup, this my well explain the absence of a supercurrent. However, the signature of a crossover towards a Josephson supercurrent is already visible in Figs. 4a, b, where we observe an enhanced suppression of the differential resistance when $|V| \lesssim 40 \mu \mathrm{V}$ and $|B| \lesssim 1 \mathrm{mT}$.

To further investigate the ZBA, we have fabricated a second array of S-TI-S junctions. The junctions have the same geometry, but the contact interfaces have been improved by a modified fabrication process. After e-beam patterning and development, we have now cleaned the HgTe surface by exposing it to a very mild, low-power, Ar plasma prior to Nb sputtering. This plasma cleaning results in more transparent $\mathrm{Nb}-\mathrm{HgTe}$ interfaces without affecting the quality of the surface states. This device has been fabricated on a similar $\mathrm{HgTe}$ layer of $70 \mathrm{~nm}$ thickness [8]. The differential resistance of this junction (see inset of Fig. 5a) has a smaller increase in the subgap region $(|V|<2 \mathrm{mV})$, indicating a smaller tunnel barrier at the interfaces. The ZBA appears at a higher bias value than in the previous sample (at $|V| \lesssim 200 \mu V$; see dotted line in Fig. 5a), consistent with a larger induced gap, as expected for more transparent contacts. Also the zero-bias value of $d V / d I$ (with respect to $R_{n}$ ) is much smaller than in the previous sample.

That we are approaching the supercurrent regime is convincingly shown by measurements of the differential resistance as function of magnetic field (Figs. 5b, c). At zero-bias, we observe an oscillating behaviour of $d V / d I$ with maxima at $B=n \Delta B$ for $n= \pm 1, \pm 2, \ldots$ (see dotted lines in Fig. 5b), and minima for $n=0, \pm 1.5, \pm 2.5, \ldots$ (with $\Delta B \approx 1.5 \mathrm{mT}$ ). This periodic behaviour is closely related to a Fraunhofer pattern that is usually observed in the supercurrent regime of a Josephson junction [10], and which shows the presence of a uniform current through proximity-induced coherent states between the two $\mathrm{Nb}$ contacts. The effective area $(A)$ can be determined from the periodicity of the $d V / d I$ oscillations, and is equal to $A=\Phi_{0} / \Delta B \approx 1.3 \mu \mathrm{m}^{2}$ (where $\Phi_{0}=h / 2 e$ is the flux quantum). The width of the $\mathrm{HgTe}$ stripe is 2 $\mu \mathrm{m}$, giving an effective length of $650 \mathrm{~nm}$, which is approximately equal to the distance between the centers of the Nb leads. This result clearly shows that a precursor of the Josephson effect occurs in the topological surface states of strained HgTe.

We thank C. Ames, P. Leubner, M. Mühlbauer, and C. Thienel for fabrication and characterization of the $\mathrm{HgTe}$ layers, and B. Trauzettel and P. Recher for useful discussions. This work was financially supported by the German research foundation DFG [SPP 1285 Halbleiter Spintronik, DFG-JST joint research program 'Topological Electronics', Emmy-Noether Grant, No. HA5893/31], and the EU ERC-AG program [project 3-TOP].

* Electronic address: jeroen.oostinga@physik.uni-wuerzburg.de

[1] M. Z. Hasan, C. L. Kane, Rev. Mod. Phys. 82, 3045 (2010); X. L. Qi, S. Z. Zhang, Rev. Mod. Phys. 83, 1057 
(2011).

[2] M. König et al., Science 318, 766 (2007).

[3] D. Hsieh et al., Nature 452, 970 (2008); Y. Xia et al., Nat. Phys. 5, 398 (2009); Y. L. Chen et al., Science 325, 178 (2009).

[4] L. Fu, C. L. Kane, Phys. Rev. Lett. 100, 096407 (2008).

[5] B. Sacépé et al., Nat. Commun. 2:575, doi: 10.1038/ncomms1586 (2011); J. R. Williams et al., Phys. Rev. Lett. 109, 056803 (2012).

[6] M. Veldhorst et al., Nat. Mater. 11, 417 (2012).

[7] C. Brüne et al., Phys. Rev. Lett. 106, 126803 (2011).

[8] See Supplemental Online Material.

[9] A. M. Shuvaev et al., arXiv: 1208.1115 (2012).

[10] M. Tinkham, Introduction to Superconductivity (McGraw-Hill, New York, 1996).

[11] M. Octavio, M. Tinkham, G. E. Blonder, T. M. Klapwijk, Phys. Rev. B 27, 6739 (1983).

[12] B. Pannetier, H. Courtois, J. Low Temp. Phys. 118, 599 (2000); T. M. Klapwijk, J. Supercond. 17, 593 (2004).

[13] T. D. Stanescu, J. D. Sau, R. M. Lutchyn, S. Das Sarma, Phys. Rev. B 81, 241310(R) (2010).

[14] A. F. Volkov, T. M. Klapwijk, Phys. Lett. A 168, 217 (1992); M. Fogelström, D. Rainer, J. A. Sauls, Phys. Rev. Lett. 79, 281 (1997); G. Tkachov, V. I. Fal'ko, Phys. Rev. B 69, 092503 (2004).

[15] F. Rohlfing et al., Phys. Rev. B 80, 220507(R) (2009).

[16] B. D. Josephson, Phys. Lett. 1, 251 (1962).

[17] P. Jarillo-Herrero, J. A. van Dam, L. P. Kouwenhoven, Nature 439, 953 (2006); H. I. Jorgensen, K. GroveRasmussen, T. Novotny, K. Flensberg, P. E. Lindelof, Phys. Rev. Lett. 96, 207003 (2006). 